\title{
Pathological and Clinicopathological Features of Canine and Feline Bladder Disease
}

\author{
Emily E. Jones ${ }^{1}$ \\ Thesis submitted for the degree of Doctor of Philosophy, \\ The University of Queensland
}

\begin{abstract}
Dogs and cats commonly present to veterinary hospitals with urinary bladder disease, but despite their clinical importance and comparative potential to human diseases, bladder diseases in Australian dogs and cats are under-investigated. In veterinary pathology, diagnostic agreement is influenced by the pathologist's own experience, training, and cognitive biases. Logistic regression is a statistical technique which has the potential to improve veterinary pathologist agreement. The main thesis goals were to investigate the pathology and comparative potential of canine and feline urinary bladder disease in Australia, and to explore the utility of logistic regression modelling in improving inter-pathologist agreement.

This project evaluated pathology cases of canine and feline bladder tissue from the veterinary pathology archives of The University of Queensland and Murdoch University, with prospective sampling from veterinary clinics and a veterinary pathology laboratory in South East Queensland. The dataset demographics and a comprehensive analysis of histological features were examined using logistic regression to identify associations between the diagnosis and animal factors and histological features, respectively. Next, a subset of canine and feline bladder tissues was tested for biomarker expression using immunohistochemistry and polymerase chain reaction. To further investigate the comparative potential of feline idiopathic cystitis (FIC), a systematic review was conducted on biomarkers in bladder pain syndrome (BPS) compared to FIC. Finally, the modelling of histological variables was used to formulate a predictive probability tool which was tested on four veterinary pathologists.

The demographic analysis showed a higher risk of bladder neoplasia in dogs compared to cats and increasing risk for bladder neoplasia with age. Next, logistic regression modelling on the histology features identified six significant variables that were associated with the diagnosis - urothelial ulceration, urothelial inflammation, neutrophilic submucosal inflammation, submucosal lymphoid aggregates, amount of submucosal haemorrhage, and species. The pathologist agreement study revealed a good level of agreement between the four pathologists when diagnosing neoplastic lesions, but poor to fair agreement for cystitis, urolithiasis and normal bladder tissue. Agreement between pathologists was variable when the predictive probability tool was used; however, using the predictive tool increased the agreement between the study pathologists' diagnosis and the reference diagnosis. A systematic review on biomarkers in bladder pain syndrome revealed that nerve growth factor is the most likely urine biomarker to be useful in the diagnosis of human BPS. Finally, investigation of biomarkers of canine and feline bladder diseases showed that tight junction protein-1 may be a promising tissue biomarker for differentiating between some urinary bladder diseases in these species.

This thesis has undertaken a comprehensive analysis of the pathogenesis and comparative potential of canine and feline bladder diseases and is the first to apply logistic regression modelling to veterinary

This work is licensed under a Creative Commons Attribution-NonCommercial-NoDerivatives 4.0 International Licence. Individual articles may be copied or downloaded for private, scholarly and not-for-profit use. Quotations may be extracted provided that the author and The Royal Society of Queensland are acknowledged. Queries regarding republication of papers, or parts of papers such as figures and photographs, should be addressed to the Secretary of The Royal Society of Queensland (rsocqld@gmail.com).
\end{abstract}


histopathology diagnosis and to improving inter-pathologist agreement. Dogs and cats are potentially good comparative models for human bladder diseases; however, inconsistent case definitions in human research complicate veterinary and medical field alignment. Finally, a collaborative multicentre approach would be invaluable to collect high quality prospective samples of feline idiopathic cystitis cases to allow further investigation into this disease.

${ }^{1}$ School of Veterinary Science, The University of Queensland, Gatton, QLD 4343 (emily.jones@uq.edu.au)

Jones, E. E. (2021). Pathological and clinicopathological features of canine and feline bladder disease [Abstract]. Proceedings of The Royal Society of Queensland, 129, x-xx. https://doi.org/10.53060/prsq.2021.a1 